\title{
CONTINUITY OF MAXIMAL MONOTONE SETS IN BANACH SPACE'
}

\author{
R. E. SHOWALTER
}

Abstract. A monotone set-valued map of a Banach space to its dual is shown to map line segments into bounded sets. It follows that convergent sequences are mapped into bounded sets and, when the space is separable or reflexive, this imposes continuity requirements on maximal monotone maps.

Let $X$ be a (real) Banach space with dual $X^{*}$ and denote the value of $w \in X^{*}$ on $x \in X$ by $(w, x)$. We consider a subset $T$ of $X \times X^{*}$ whose image $T(x)=\left\{w \in X^{*}:[x, w] \in T\right\}$ at each $x \in X$ is nonempty. The set $T$ is called monotone if

$$
\left(w_{1}-w_{2}, x_{1}-x_{2}\right) \geqq 0, \quad\left[\dot{x}_{1}, w_{1}\right],\left[x_{2}, w_{2}\right] \in T,
$$

and maximal monotone if it is not properly included in a monotone set. We give an elementary proof that monotone sets map line segments into bounded sets and then deduce some continuity properties of maximal monotone sets.

THEOREM 1. Let $T$ be a monotone subset of $X \times X^{*}$ with nonempty image $T(x)$ for each $x \in X$. Let $x_{0}$ and $x_{1}$ belong to $X$, and set $x_{t}=x_{0}+$ $t\left(x_{1}-x_{0}\right)$ for $t \in[0,1)$. Then $\bigcup\left\{T\left(x_{t}\right): t \in[0,1)\right\}$ is bounded in $X^{*}$.

Proof. Let $v \in X$ and choose $w_{1} \in T\left(x_{1}\right), w_{2} \in T\left(x_{0}+v\right)$. For each $u \in T\left(x_{t}\right)$ we have by monotonicity of $T$

$$
\left(w_{1}-u, x_{1}-x_{t}\right)=(1-t)\left(w_{1}-u, x_{1}-x_{0}\right) \geqq 0,
$$

so we obtain $\left(u, x_{1}-x_{0}\right) \leqq\left(w_{1}, x_{1}-x_{0}\right)$. The monotonicity also gives us

$$
\begin{aligned}
(u, v) & \leqq t\left(u, x_{1}-x_{0}\right)+\left(w_{2}, v-t\left(x_{1}-x_{0}\right)\right) \\
& \leqq\left|\left(w_{1}, x_{1}-x_{0}\right)\right|+\left(w_{2}, v\right)+\left|\left(w_{2}, x_{1}-x_{0}\right)\right| .
\end{aligned}
$$

Repeating the argument with $v$ replaced by $-v$ shows that $(u, v)$ is bounded independently of $t \in[0,1)$ and $u \in T\left(x_{t}\right)$, so the desired result follows by uniform boundedness.

Received by the editors March 19, 1973 and, in revised form, May 2, 1973.

AMS (MOS) subject classifications (1970). Primary 47H05; Secondary 47H15.

${ }^{1}$ Research supported in part by National Science Foundation grant GP-34261.

(C) American Mathematical Society 1974 
THEOREM 2. Let $T$ be a monotone set with $T(x)$ nonempty for each $x \in X$. If $\left\{x_{n}\right\}$ is a convergent sequence in $X$, then $\bigcup\left\{T\left(x_{n}\right): n \geqq 1\right\}$ is bounded in $X^{*}$.

Proof. Since $T\left(x_{n}\right)$ is bounded for each $n \geqq 1$ by Theorem 1 , it suffices to show that for any choice of $v_{n} \in T\left(x_{n}\right)$ for each $n$, the sequence $\left\{v_{n}\right\}$ is bounded. But this will follow from the proof in [3].

REMARK. The writer was informed by the referee and by L. Nirenberg of the references [4], [5] which contain Theorem 2. The simple proof of Theorem 1 seems to be new, and it extends easily to the case where the domain $D(T)=\{x \in X: T(x)$ is nonempty $\}$ is not all of $X$. One needs then to require that $x_{0}$ belong to the interior of $D(T)$ and $x_{1} \in D(T)$. Theorem 2 holds if $\left\{x_{n}\right\}$ converges to a point in the interior of $D(T)$.

A subset of $X \times X^{*}$ is called vaguely continuous [1] if for each pair $x_{0}, x_{1} \in X$ there exists a null sequence of positive real numbers $\left\{t_{n}: n \geqq 1\right\}$, a sequence $v_{n} \in T\left(x_{n}\right)$, where $x_{n}=x_{0}+t_{n}\left(x_{1}-x_{0}\right)$, and a $v_{0} \in T\left(x_{0}\right)$ such that $\left\{v_{n}\right\}$ is weak* convergent to $v_{0}$. We define $T$ to be hemicontinuous if, for each pair $x_{0}, x_{1} \in X$, for every null sequence of positive real numbers $\left\{t_{n}\right\}$, and for every choice of $v_{n} \in T\left(x_{n}\right), x_{n}$ given as above, there exists a subsequence $\left\{v_{n}^{\prime}\right\}$ which is weak* convergent to some $v_{0} \in T\left(x_{0}\right)$. Finally, we define $T$ to be demicontinuous if for every sequence $\left\{x_{n}\right\}$ which converges to $x_{0} \in X$, and for every choice of $v_{n} \in T\left(x_{n}\right)$, there exists a subsequence $\left\{v_{n}^{\prime}\right\}$ which is weak* convergent to some $v_{0} \in T\left(x_{0}\right)$.

It is clear that each of these conditions implies the preceding one. Also, if $T\left(x_{0}\right)=\left\{v_{0}\right\}$ is a singleton in the definition of either hemicontinuity or demicontinuity, then the original sequence $\left\{v_{n}\right\}$ converges to $v_{0}$. Hence, if $T$ is a function, these definitions agree with the usual notions of hemicontinuity and demicontinuity [3].

It is known [1, Theorem 1.2] that if $T$ is monotone, vaguely continuous, and if $T(x)$ is closed and convex for each $x \in X$, then $T$ is maximal monotone. Conversely [1, Lemma 1.1], if $T$ is maximal monotone it follows that $T(x)$ is closed and convex for each $x \in X$. The converse is complete when the closed unit ball of $X^{*}$ is weak* sequentially compact.

THEOREM 3. Let $X$ be separable or reflexive. A maximal monotone set $T$ with $T(x)$ nonempty for each $x \in X$ is demicontinuous.

Proof. Let $\left\{x_{n}\right\}$ converge to $x_{0}$ in $X$ and $v_{n} \in T\left(x_{n}\right)$. Theorem 2 asserts that $\left\{v_{n}\right\}$ is bounded, so by weak* compactness of the unit ball of $X^{*}$, it suffices to show that if $\left\{v_{n}\right\}$ is weak* convergent to $v_{0}$, then $v_{0} \in T\left(x_{0}\right)$. But for each $x \in X$ and $w \in T(x)$ we have by monotonicity

$$
\left(v_{n}-w, x_{n}-x\right) \geqq 0, \quad n \geqq 1,
$$


so taking the limit gives

$$
\left(v_{0}-w, x_{0}-x\right) \geqq 0, \quad[w, x] \in T .
$$

Since $T$ is maximal monotone, $v_{0} \in T\left(x_{0}\right)$.

COROLlaRY 1. Let $X$ be separable or reflexive. The set $T$ is maximal monotone if and only if it is monotone, vaguely continuous, and $T(x)$ is closed and convex for each $x \in X$.

Clearly, Corollary 1 is true with vague continuity replaced by hemicontinuity or demicontinuity (or upper semicontinuity [2]) so these notions agree for monotone sets whose images are closed and convex. Furthermore, since the sum

$$
T_{1}+T_{2}=\left\{\left[x, w_{1}+w_{2}\right]:\left[x, w_{j}\right] \in T_{j}, j=1,2\right\}
$$

of a vaguely continuous set $T_{1}$ and a hemicontinuous set $T_{2}$ is vaguely continuous, we have a perturbation result.

Corollary 2. Let $T_{1}$ be vaguely continuous and $X$ be separable or reflexive. If $T_{2}$ is maximal monotone and the sum $T_{3}=T_{1}+T_{2}$ is monotone with $T_{3}(x)$ closed, convex and nonempty for each $x \in X$, then $T_{3}$ is maximal monotone. In particular, if $T_{2}$ and $T_{3}$ are monotone with $T_{2}(x)$ and $T_{3}(x)$ closed, convex and nonempty for each $x \in X$, and if $T_{1}$ is vaguely continuous and single valued, then $T_{2}$ is maximal monotone if and only if $T_{3}$ is maximal monotone.

Finally, if the range of $T, \bigcup\{T(x): x \in X\}$ is all of $X^{*}$ and $X$ is reflexive, then the inverse set $T^{-1}=\{[x, w]:[w, x] \in T\}$ is monotone (maximal monotone) if and only if $T$ is monotone (respectively, maximal monotone). This gives

Corollary 3. Let $T$ be a monotone set in $X \times X^{*}$ with $X$ reflexive. Suppose that $T(x)$ and $T^{-1}(w)$ are closed, convex and nonempty for each $x \in X$ and $w \in X^{*}$. Then $T$ is vaguely continuous (or hemicontinuous or demicontinuous) if and only if $T^{-1}$ is vaguely continuous (or hemicontinuous or demicontinuous).

The preceding results give improvements in many of the results of [1], [2]: we obtain converses to certain of these, and we may drop boundedness assumptions in others. Theorem 2 shows that monotone sets map compact sets into bounded sets, so if $X$ has finite dimension, a monotone $T$ maps bounded sets to bounded sets and is maximal if and only if it is continuous. 


\section{REFERENCES}

1. F. E. Browder, Multivalued monotone nonlinear mappings and duality mappings in Banach spaces, Trans. Amer. Math. Soc. 118 (1965), 338-351. MR 31 \#5114.

2. - Nonlinear variational inequalities and maximal monotone mappings in Banach spaces, Math. Ann. 183 (1969), 213-231. MR 42 \#6661.

3. T. Kato, Demicontinuity, hemicontinuity and monotonicity. II, Bull. Amer. Math. Soc. 73 (1967), 886-889. MR 38 \#6411.

4. P. M. Fitzpatrick, P. Hess and T. Kato, Local boundedness of monotone-type operators, Proc. Japan Acad. 48 (1972), 275-277.

5. R. T. Rockafellar, Local boundedness of nonlinear, monotone operators, Michigan Math. J. 16 (1969), 397-407. MR 40 \#6229.

Department of Mathematics, University of TeXas, Austin, Texas 78712 